Frontiers in Heat and Mass Transfer

\title{
EFFECT OF TEMPERATURE ON WATER TRANSPORTATION IN NANOCHANNEL
}

\author{
Yongbin Zhang* \\ College of Mechanical Engineering, Changzhou University, Changzhou, 213164, Jiangsu Province, China
}

\begin{abstract}
The flow factor approach model was used to study the effect of temperature on water transportation in a nano slit pore flow driven by the pressure. The influences of the temperature on the density and viscosity of water and on the water-wall interaction were considered. The results show that enhancing the temperature of water significantly improves water transportation in nanochannel, especially when the channel height is so low that the water non-continuum effect is significant. The mechanism of this temperature effect is that the temperature increase not only appreciably reduces the water viscosity but also considerably alleviates the water non-continuum effect, in these circumstances the water flow rate through the channel owing to the Poiseuille flow is significantly increased.
\end{abstract}

Keywords: Water transportation; Nanochannel; Flow; Temperature; Model

\section{INTRODUCTION}

The super-purification of water is important to the life of human beings. It needs to filter out very small substances such as bacteria, virus, tiny pollutants, organic contaminants and ions. It often relies on the use of nanoporous filtration membrane, which prevents the going-through of big size molecules but permit water to flow through (Das, et al., 2014; Han et al., 2013; Jackson and Hillmyer, 2010; Surwade, et al., 2015; Wei, et al., 2014). The flow of water in a nanoporous membrane is driven by the pressure, and this flow rate is very small because of the confinement of water in the nanoscale channel (Gordillo and Marti, 2007; Marti, et al., 2010; Marti, et al.,2006; Mamontov, et al., 2005; Nagy, et al., 2007; Su and Guo, 2012; Zangi, 2004; Zhang, 2015). It is a research task that how to improve the flow of water through a nanochannel. This research is definitely of significant interest to the treatment of water by nanofluidics.

For improving the transportation, the concept of the nanotube tree with branches was proposed (Zhang, 2017). It was suggested that the transportation capacity of the nanotube tree is equivalent to that of a conventional tube the inner radius of which is on the millimeter scale even when the fluid-wall interaction is strong (Zhang, 2017).

On the other hand, from the principle of the Poiseuille flow in a nanochannel, we can explore the way to improve the transportation capacity of a single nanochannel. According to the flow factor approach model for nanochannel flow (Zhang, 2016), it was found that the mass flow rate through the nanochannel owing to the Poiseuille flow is largely influenced by the effective viscosity of the confined fluid and the fluid non-continuum effect, both of which are enhanced with the reduction of the channel height and actually strongly impedes the fluid flow through the channel. These two factors are however strongly influenced by the fluid temperature, the increase of which remarkably reduces the fluid viscosity, the interaction strength between the fluid and the wall and thus the fluid non-continuum effect. The enhancement of the fluid temperature thus should have a significant positive effect on mass transportation through a nanochannel.

The present paper carries out an analysis for the effect of water temperature on the transportation of water through the nanochannel owing to the Poiseuille flow, by using the flow factor approach model. The water temperature varies from $5^{\circ} \mathrm{C}$ to $90{ }^{\circ} \mathrm{C}$. The temperature effect for different channel heights was investigated. The calculation results show that enhancing the water temperature greatly improves the transportation capacity of water through the nanochannel, especially when the channel height is low.

\section{ANALYSIS}

The mass flow rate of water through a nano slit pore driven by the pressure is analytically investigated by using the flow factor approach model. The influences of the pressure of the confined water on the density and viscosity of water in the channel are neglected, as the pressure in nanofluidics is normally not so high. For convenience, the water film slippage at the wall surface is also neglected; This should not alter the obtained conclusions.

For no interfacial slippage, the mass flow rate per unit channel length through a nano slit pore owing to the Poiseuille flow is (Zhang, 2016):

$q_{m, b f}=\frac{S \rho_{b f}^{e f f} h^{3}}{12 \eta_{b f}^{e f f}} \frac{d p}{d x}$

where $h$ is the channel height, $p$ is the pressure of the confined fluid, $x$ is the coordinate along the flow direction, $\rho_{b f}^{e f f}, \eta_{b f}^{e f f}$ and $S$ are respectively the average density of the fluid across the channel height, the effective viscosity of the fluid and the parameter accounting for the discontinuity and inhomogeneity effects of the fluid across the channel height i.e. the non-continuum effect of the fluid $(-1 \leq S<0)$. It is defined that:

$r=\left|\frac{12 \eta_{1} q_{m, b f}}{\rho_{1} h^{3} d p / d x}\right|$

*Email: engmech1@sina.com 
where $\rho_{1}$ and $\eta_{1}$ are respectively the bulk density and bulk viscosity of the fluid for a reference high temperature $T_{1}$ under ambient pressure. The parameter $r$ represents the ratio of $q_{m, b f}$ to the mass flow rate per unit channel length through the channel at the high temperature $T_{1}$ based on the continuum fluid assumption. It can be used to measure the mass transportation ability of the channel at different temperatures.

The parameter $\rho_{b f}^{e f f}$ at low pressures is formulated as dependent on the fluid temperature and the channel height and it is (Zhang, 2017) :

$\rho_{b f}^{e f f}(T, h)=\rho_{1}\left[1-k_{\rho}\left(T-T_{1}\right)\right] C q(T, h)$

where $T$ is the fluid temperature in ${ }^{o} C, T_{1}$ is a reference high temperature, $\rho_{1}$ is the bulk density of the fluid at the temperature $T_{1}$ under low pressure, $k_{\rho}$ is constant, and $C q$ is the parameter accounting for the fluid solidification due to the confinement of the wall.

The parameter $\eta_{b f}^{e f f}$ at low pressures is also formulated as dependent on the fluid temperature and the channel height and it is (Zhang, 2017):

$\eta_{b f}^{e f f}(T, h)=\eta_{1} e^{-\lambda\left(T-T_{1}\right)} C y(T, h)$

where $\lambda$ is constant, $\eta_{1}$ is the bulk viscosity of the fluid at the temperature $T_{1}$ under low pressure, $C y$ is the parameter accounting for the wall confinement effect on the fluid viscosity.

Substituting Eq. (3) and (4) into Eq. (1) and further substituting Eq. (1) into Eq. (2) yields:

$r=\frac{|S| C q(T, h) e^{\lambda\left(T-T_{1}\right)}\left[1-k_{\rho}\left(T-T_{1}\right)\right]}{C y(T, h)}$

\section{CALCULATION}

Here, exemplary calculations were made. In the calculation, $C q(T, h)$ is interpolated according to the values of $C q\left(T_{l}, h\right)$ and $C q\left(T_{0}, h\right)$ and expressed as:

$C q(T, h)=\frac{C q\left(T_{1}, h\right)-C q\left(T_{0}, h\right)}{T_{1}-T_{0}}\left(T-T_{0}\right)+C q\left(T_{0}, h\right)$

where $C q\left(T_{1}, h\right)$ and $C q\left(T_{0}, h\right)$ are respectively the values of $C q(T, h)$ at the temperatures $T_{1}$ and $T_{0}$ which respectively correspond to weak and medium-level fluid-wall interactions. $C q\left(T_{1}, h\right)$ and $C q\left(T_{0}, h\right)$ are generally expressed as (Zhang, 2017):

$C q(H)=\left\{\begin{array}{l}1 \quad \text { for } \quad H \geq 1 \\ m_{0}+m_{1} H+m_{2} H^{2}+m_{3} H^{3}, \text { for } 0<H<1\end{array}\right.$

where $H=h / h_{c r, b f}, h_{c r, b f}$ is the critical channel height for the confined fluid to be continuum, and the values of $m_{0}, m_{1}, m_{2}$, and $m_{3}$ respectively for $T_{l}$ and $T_{0}$ are shown in Table 1(a).

$C y(T, h)$ is interpolated as:
$C y(T, h)=\frac{C y\left(T_{1}, h\right)-C y\left(T_{0}, h\right)}{T_{1}-T_{0}}\left(T-T_{0}\right)+C y\left(T_{0}, h\right)$

where $C y\left(T_{1}, h\right)$ and $C y\left(T_{0}, h\right)$ are respectively the values of $C y(T, h)$ at the temperatures $T_{1}$ and $T_{0}$ which respectively correspond to weak and medium-level fluid-wall interactions. $C y\left(T_{l}, h\right)$ and $C y\left(T_{0}, h\right)$ are generally expressed as (Zhang, 2017):

$C y(H)= \begin{cases}1 \quad, \quad \text { for } \quad H \geq 1 \\ a_{0}+\frac{a_{1}}{H}+\frac{a_{2}}{H^{2}} \quad, \quad \text { for } 0<H<1\end{cases}$

where $a_{0}, a_{1}$ and $a_{2}$ are respectively constants. The values of $a_{0}$, $a_{1}$ and $a_{2}$ respectively for $T_{1}$ and $T_{0}$ are shown in Table 1(b).

The value of $S$ is interpolated as:

$S(T, h)=\frac{S\left(T_{1}, h\right)-S\left(T_{0}, h\right)}{T_{1}-T_{0}}\left(T-T_{0}\right)+S\left(T_{0}, h\right)$

where $S\left(T_{1}, h\right)$ and $S\left(T_{0}, h\right)$ are respectively the values of $S(T, h)$ at the temperatures $T_{1}$ and $T_{0}$ which respectively correspond to weak and medium-level fluid-wall interactions. $S\left(T_{l}, h\right)$ and $S\left(T_{0}, h\right)$ are generally expressed as (Zhang, 2017):

$S(H)= \begin{cases}-1 \quad, \quad \text { for } \quad H \geq 1 \\ {\left[n_{0}+n_{1}\left(H-n_{3}\right)^{n_{2}}\right]^{-1}, \text { for } n_{3}<H<1}\end{cases}$

where $n_{0}, n_{1}, n_{2}$ and $n_{3}$ are respectively constants. The values of $n_{0}, n_{1}, n_{2}$ and $n_{3}$ respectively for $T_{1}$ and $T_{0}$ are shown in Table 1(c).

Table 1(a) Fluid viscosity data for different temperatures (Zhang, 2017)

\begin{tabular}{|c|l|l|l|}
\hline Pemperature & \multicolumn{1}{|c|}{$\mathrm{a}_{0}$} & $\mathrm{a}_{1}$ & $\mathrm{a}_{2}$ \\
\hline$T_{0}$ & & & \\
\hline$T_{l}$ & 1.0822 & -0.1758 & 0.0936 \\
\hline
\end{tabular}

Table 1(b) Fluid density data for different temperatures (Zhang, 2017)

\begin{tabular}{|c|c|c|c|c|}
\hline $\mathrm{T}_{\text {Temperature }}^{\text {Parameter }}$ & $\mathrm{m}_{0}$ & $\mathrm{~m}_{1}$ & $\mathrm{~m}_{2}$ & $\overline{m_{3}}$ \\
\hline$\overline{T_{0}}$ & 1.30 & -1.065 & 1.336 & -0.571 \\
\hline$T_{1}$ & 1.116 & -0.328 & 0.253 & -0.041 \\
\hline
\end{tabular}

Table 1(c) Fluid non-continuum property data for different temperatures (Zhang, 2017)

\begin{tabular}{|c|c|c|c|c|}
\hline Temperature & $\mathrm{n}_{0}$ & $\mathrm{n}_{1}$ & $\mathrm{n}_{2}$ & $\mathrm{n}_{3}$ \\
\hline$\overline{T_{0}}$ & -0.649 & -0.343 & -0.665 & 0.035 \\
\hline$T_{1}$ & -0.1 & -0.892 & -0.084 & 0.1 \\
\hline
\end{tabular}

\section{RESULTS}

In the calculation, the modeled fluid was water and the following parameter values were taken: 
$T_{0}=3^{\circ} \mathrm{C}, T_{1}=95^{\circ} \mathrm{C}, k_{\rho}=0.00053 /{ }^{\circ} \mathrm{C}, \lambda=0.021 /^{\circ} \mathrm{C}, h_{c r, b f}=3 \mathrm{~nm}$

for $T=T_{1}$, and $h_{c r, b f}=6 \mathrm{~nm}$ for $T=T_{0}$

The results were computed for $T$ ranging between $T_{0}$ and $T_{l}$. In this temperature range, when $h \geq 6 \mathrm{~nm}$, the channel flow is a continuum flow, and Eq. (5) reduces to:

$r=e^{\lambda\left(T-T_{1}\right)}\left[1-k_{\rho}\left(T-T_{1}\right)\right] \quad$ for a continuum flow

Figure 1 plots the values of $r$ against the temperature $T$ for different channel heights for which the confined water is in a noncontinuum flow. The obtained $r$ values are also compared with those for a continuum flow at different temperatures calculated from Eq. (12). It is shown that whenever the water is in continuum or non-continuum flows, the temperature enhancement significantly increases the value of $r$ and thus significantly increases the mass flow rate through the channel. This effect appears more pronounced for a lower $h$ for which the non-continuum effect of the water is stronger. The calculation results indicate that enhancing the fluid temperature is very helpful for transportation of the fluid through a nanochannel by the pressure driving. This may have an important engineering application value for mass and heat transfer by nanochannel, as the flow rate through a nanochannel driven by the pressure is usually very small, unavoidably limited by the strong non-continuum and viscous effects of the fluid in such a small gap .

If the interfacial slippage at the wall surface is further considered, the drawn conclusion should still stand, as the temperature rise actually stimulates the interfacial slippage and thus further increases the mass flow rate through the channel, by dropping the fluid-wall interfacial shear strength (Zhang, 2015b).

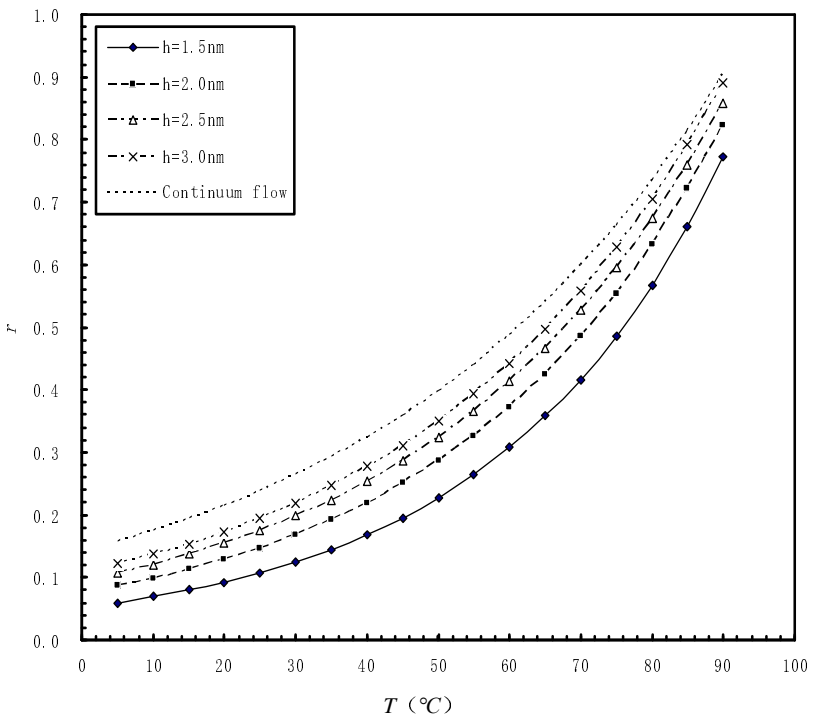

Fig. 1 Plot of the value of $r$ against the temperature $T$ for different channel heights for the confined water flow.

\section{CONCLUSIONS}

This paper analytically investigates the influence of the water temperature on the mass transportation of water in a nano slit pore driven by the pressure, by using the flow factor approach model. In the analysis, the temperature of water varied from $5{ }^{\circ} \mathrm{C}$ to $90^{\circ} \mathrm{C}$. The dependence of both the density and viscosity of the water on the temperature and the channel height were considered. The influence of the water temperature on the water-wall interaction was also incorporated.
The calculation results show that enhancing the water temperature significantly increases the water mass flow rate through the nanochannel. This effect is due to that the water temperature rise not only significantly reduces the water viscosity, but also considerably alleviates the non-continuum effect of the confined water; The latter two factors are actually harmful for the mass transportation in a nanochannel driven by the pressure.

The obtained results may have an important engineering implication by enhancing the water temperature to improve the water transportation in a nanochannel.

\section{REFERENCES}

Das, R., Eaqub Ali, Md, Abd Hamid, S. B., Ramakrishna, S., and Chowdhury, Z. Z., 2014, "Carbon Nanotube Membranes for Water Purification: A Bright Future in Water Desalination," Desalination, 336, 97-109.

https://doi.org/10.1016/j.desal.2013.12.026

Gordillo, M. C., and Marti, J., 2007, "High Temperature Behavior of Water Inside Flat Graphite Nanochannels," Physical Review B, 75, 085406.

https://doi.org/10.1103/PhysRevB.75.085406

Han, Y., Xu, Z., and Gao, C., 2013, "Ultrathin Graphene Nanofiltration Membrane for Water Purification," Advanced Functional Materials, 23, 3693-3700.

https://doi.org/10.1002/adfm.201202601

Jackson, E. A., and Hillmyer, M. A., 2010, "Nanoporous Membranes Derived from Block Copolymers: From Drug Delivery to Water Filtration," ACS Nano, 4, 3548-3553.

https://doi.org/10.1021/nn1014006

Mamontov, E., Kumzerov, Yu A., and Vakhrushev, S. B., 2005, "Translational Dynamics of Water in the Nanochannels of iented Chrysotile Asbestos Fibers," Physical Review E, 71,061502.

https://doi.org/10.1103/PhysRevE.71.061502

Marti, J., Sala, J., and Guardia, E., 2010, "Molecular Dynamics Simulations of Water Confined in Graphene Nanochannels: From Ambient to Supercritical Environments," Journal of Molecular Liquids, 153, 72-78.

https://doi.org/10.1016/j.molliq.2009.09.015

Marti, J., Nagy, G., Gordillo, M. C., and Guardia, E., 2006, "Molecular Simulation of Lquid Water Confined Inside Graphite Channels: Thermodynamics and Structural Properties," Journal of Chemical Physics, 124, 094703.

https://doi.org/10.1063/1.2172590

Nagy, G., Gordillo, M. C., Guardia, E., and Marti, J., 2007, "Liquid Water Confined in Carbon Nanochannels at High Temperatures," Journal of Physical Chemistry, 111, 12524-12530. https://doi.org/10.1021/jp073193m

Surwade, S. P., Smirnov, S. N., Vlassiouk, I. V., Unocic, R. R., Veith, G. M., Dai, S., and Mahurin, S. M., 2015, "Water desalination using nanoporous single-layer grapheme," Nature Nanotechnology, 10, 459464.

https://doi.org/10.1038/nnano.2015.37

Su, J., and Guo, H., 2012, "Effect of Nanochannel Dimension on the Transport of Water Molecules," Journal of Physical Chemistry, 116, 5925-5932. https://doi.org/10.1021/jp211650s

Wei, N., Peng, X., and Xu, Z., 2014, "Understanding Water Permeation in Graphene Oxide Membranes," ACS Applied Materials and Interfaces, 6, 5877-5883.

https://doi.org/10.1021/am500777b 
Zangi, R., 2004, "Water Confined to a Slab Geometry: A Review of Recent Computer Simulation Studies," Journal of Physics: Condensed Matter, 16, 5371-5388.

https://doi.org/10.1088/0953-8984/16/45/005

Zhang, Y. B., 2015, “The Flow Factor Approach Model for the Fluid Flow in a Nano Channel," International Journal of Heat and Mass Transfer, 89, 733-742.

https://doi.org/10.1016/j.ijheatmasstransfer.2015.05.092
Zhang, Y. B., 2016, "The Flow Equation for a Nanoscale Fluid Flow," International Journal of Heat and Mass Transfer, 92, 1004-1008. https://doi.org/10.1016/j.ijheatmasstransfer.2015.09.008

Zhang, Y. B., 2017, “Transport in a Nanotube Tree,” International Journal of Heat and Mass Transfer, 114, 536-540. https://doi.org/10.1016/j.ijheatmasstransfer.2017.06.105 